\title{
Simulation numérique du transport sédimentaire dans l'estuaire de la Seine avec un modèle diphasique 2-DV
}

\author{
J. Chauchat(a), S. Guillou(b), N.V. Phan(c), N. Barbry (d)et K.D. Nguyen(d)
}

\author{
a Laboratoire de Morphodynamique Continentale et Côtire CNRS UPRESA-6143 \\ Université de Caen 24, rue des Tilleuls, 14000 CAEN, France \\ ${ }^{b}$ LUSAC, Site Universitaire, BP 78, 50130 Octeville,France \\ ${ }^{c}$ Institute of Mechanics, National Center for Natural Sciences and Technologie, \\ 264 Doican, Hanoi, Vietnam \\ 'IUT Génie Civil Saint Nazaire 58, rue Michel Ange, \\ BP 420, 44606 Saint Nazaire, France
}

\section{Résumé :}

Cet article présente des résultats préliminaires de simulation numérique du déplacement du bouchon vaseux dans l'estuaire de la Seine par un modèle à diphasique bidimensionnel vertical. Dans ce modèle, un maillage eulérien adaptatif a été utilisé. Dans un premier temps, la turbulence est parametrisée par un modèle à zéro équation de type "longueur de mélange". Les résultats de ce modèle sont en accord avec des études précédentes et le déplacement du bouchon vaseux est bien reproduit.

\section{Summary :}

The paper presents the numerical results, using a 2-D (XZ) two-phase (fluid and solid phases) model in order to simulate the displacement of the turbidity maximum in the Seine estuary. In this model, a sigma coordinates transformation has been used and the turbulence is parametrized by a zero equation model. The computational results are in good agreement with the observation and some previous studies. In particular, the displacement of the turbidity maximum has been reproduced reasonably.

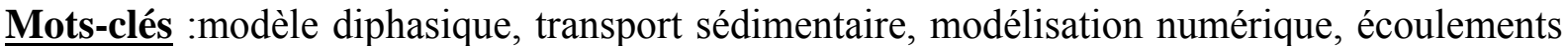
estuariens

\section{Introduction}

La plupart des modèles numériques hydrosédimentaires utilisés actuellement sont basés sur l'hypothèse simplifiée selon laquelle les particules en suspension se déplacent à la même vitesse que les particules fluides, exception faite de leur vitesse de chute. C'est l'hypothèse dite du "scalaire passif" pour la phase solide. L'impact du mouvement des particules sur l'état d'écoulement du fluide et de la turbulence est alors complètement ignoré ainsi que les interactions particule-particule qui peuvent être importantes lorsque celles-ci sont nombreuses. Une difficulté supplémentaire vient de la définition du fond, en effet, on définit un fond fictif lorsque la concentration de particules solides dépasse un seuil (souvent fixé à 20 $\mathrm{g} / \mathrm{L})$. En-dessous du fond fictif, se trouve la "crème de vase" dont l'épaisseur varie de 0.2 à 2 mètres selon les estuaires. Cette couche est considérée comme un réservoir de particules solides dans lequel se produisent les processus de tassement et de consolidation. Ces processus ne sont actuellement modélisés que sur la verticale par des formules empiriques.

La modélisation à deux phases consiste à adopter une vision globale du domaine, le domaine physique integrerait le domaine fluide et le domaine à forte teneur en sédiments (vase) avec le même type d'équation. Potentiellement, les phénomènes à l'origine du depôt, de l'érosion et du tassement y sont représentés par des lois comme la loi de Darcy intégrées directement dans les équations de transports. 
L'objet de cette communication est de présenter des résultats de simulation du deplacement du bouchon vaseux dans l'estuaire de la Seine en utilisant une approche à deux phases. Pour ce faire nous utilisons un modèle diphasique présenté par Barbry et al. [1] et adapté à la Seine.

\section{Description mathématiques du modèle}

Dans l'approche à deux phases (fluide - particule solide) on considère que les mouvements de chaque phase sont régis respectivement par deux équations de conservation de la masse et de la quantité de mouvement. Ainsi en notant k, l'indice désignant la phase fluide ou la phase solide, les équations de conservation sont données par (1) et (2), respectivement.

$$
\begin{aligned}
& \frac{\partial\left(\alpha_{k} \rho_{k}\right)}{\partial t}+\nabla \cdot\left(\alpha_{k} \rho_{k} \mathbf{u}_{\mathbf{k}}\right)=0 \\
& \frac{\partial\left(\alpha_{k} \rho_{k} \mathbf{u}_{\mathbf{k}}\right)}{\partial t}+\nabla \cdot\left(\alpha_{k} \rho_{k} \mathbf{u}_{\mathbf{k}} \wedge \mathbf{u}_{\mathbf{k}}\right)=\nabla \cdot\left(\alpha_{\mathbf{k}}\left(\overline{\overline{\mathrm{T}}}_{\mathrm{k}}^{\mathrm{Re}}+\overline{\overline{\mathrm{T}}}_{\mathrm{k}}^{\mathrm{f}}\right)\right)+\alpha_{\mathrm{k}} \rho_{\mathrm{k}} \mathbf{g}+\mathbf{M}_{\mathbf{k}}
\end{aligned}
$$

Dans ces équations, $\alpha_{k}, \mathbf{u}_{\mathbf{k}}, \rho_{k}$ représentent respectivement la fraction volumique, le vecteur vitesse et la masse volumique de la phase $\mathrm{k}$. $\mathbf{g}$ est le vecteur accélération de la Pesanteur. $\overline{\bar{T}}_{k}^{\mathrm{Re}}$ représente le tenseur des contraintes de Reynolds et $\overline{\bar{T}}_{k}^{f}$ le tenseur des contraintes de frottement qui est donné en fonction de la pression $p_{k}$ et du déviateur des contraintes $\bar{\tau}_{k}$ (contrainte visqueuse) par la relation (3).

$$
\overline{\bar{T}}_{k}^{f}=-p_{k} \overline{\bar{I}}+\overline{\bar{\tau}}_{k}
$$

Le terme $\mathbf{M}_{\mathbf{k}}$ représente les échanges de quantité de mouvement entre les deux phases. Il est évalué par la relation (4) en fonction de la pression et de la contrainte interfaciale $p_{k i}$ et $\bar{\tau}_{k i}$ et de $\mathbf{M}_{\mathbf{k}}^{\prime}$ qui représente les différentes forces agissant sur la phase $\mathrm{k}$ dont la force de traînée.

$$
\mathbf{M}_{\mathbf{k}}=\mathrm{p}_{\mathrm{ki}} \nabla \alpha_{\mathrm{k}}-\overline{\bar{\tau}}_{\mathrm{ki}} \nabla \alpha_{\mathrm{k}}+\mathbf{M}_{\mathbf{k}}^{\prime}
$$

Nous particularisons ces équations par la suite en remplaçant l'indice $\mathrm{k}$ par $f$ pour désigner les grandeurs associées à la phase fluide et par $s$ pour désigner les grandeurs associées à la phase solide. Notons que $\alpha_{s}+\alpha_{f}=1$.

On considère que la pression de la phase solide à l'interface fluide-solide est donnée par l'équation de Laplace $\left(p_{s i}=p_{f i}+H \cdot \sigma_{p}\right)$ en fonction de la tension de surface $\sigma_{p}$ et du paramètre $H=4 / d$ (où $d$ est le diamètre des particules), ainsi que la pression interfaciale du fluide $p_{f i}$. Ce dernier terme est évalué en fonction des caractéristiques de l'écoulement par : $p_{f i}=p_{f}-1 / 4 \rho_{f}\left|\mathbf{u}_{\mathbf{f}}-\mathbf{u}_{\mathrm{s}}\right|$. Le gradient de pression dans la phase particulaire est considéré comme la somme de deux effets $: \nabla\left(\alpha_{s} p_{s}\right)=\nabla\left(\alpha_{s} p_{f}\right)+\nabla\left(\alpha_{s} p_{s, \text { coll }}\right)$. Le terme de pression collisionnelle, dû aux interactions entre les particules, a été modélisé de la façon suivante : $\nabla\left(\alpha_{s} p_{s, \text { coll }}\right)=-G\left(\alpha_{f}\right) \cdot \nabla\left(\alpha_{s}\right)$ avec $G\left(\alpha_{f}\right)=G_{0} e^{-C \cdot\left(\alpha_{f}-\alpha^{*}\right)}$ où $\mathrm{G}_{0}, \mathrm{C}$ et $\alpha^{*}$ sont des constantes, nous avons utilisé les valeurs proposées par Lyczkowski et al. [2]. 
Le tenseur de cisaillement de la phase $k$ à l'interface a été calculé par $\stackrel{=}{=}{ }_{s i}=\bar{\tau}_{f i}=\beta \cdot{ }_{\bar{\tau}}$. Le paramètre $\beta$ prend en compte l'interaction entre les particules. Il est fonction de la distance interparticulaire $h$ et du diamètre $d$ des particules. La formulation retenue est celle proposée par Graham (1981) [3].

$\mathbf{M}_{\mathrm{s}}^{\prime}$ est la somme des forces de traînée, des forces de masse virtuelle, des forces ascentionnelles. Pour le fluide $: \mathbf{M}_{\mathrm{f}}^{\prime}=-\mathbf{M}_{\mathrm{s}}^{\prime}$. Pour le moment, seule la force de traînée est prise en compte :

$$
\mathbf{F}_{\mathbf{D}}=\frac{3}{4} \frac{\alpha_{\mathrm{s}} \rho_{\mathrm{f}} \mathrm{C}_{\mathrm{D}}}{\mathrm{d}}\left|\mathbf{u}_{\mathbf{f}}-\mathbf{u}_{\mathbf{s}}\right|\left(\mathbf{u}_{\mathbf{f}}-\mathbf{u}_{\mathbf{s}}\right)
$$

Le coefficient de traînée $\left(C_{D}\right)$ est fonction du nombre de Reynolds particulaire et de la fraction volumique de la phase particulaire $\alpha_{s}$ [4].

Les conditions aux limites sont imposées de la manière suivante :

- A la surface libre, deux conditions sont imposées, une condition dynamique qui assure la continuité de la pression du fluide à l'interface et une condition cinématique qui consiste à considérer la surface libre comme une ligne de courant.

- Sur le fond, une condition de réflexion sur la pression du fluide et une condition d'adhérence sur la vitesse du mélange sont imposées. Le fond est imperméable aux sédiments.

- A la frontière Ouest (côté mer), on impose une condition de marée (élévation imposée). La fraction volumique de sédiment est calculé par le modèle dans le cas d'un écoulement sortant et en imposant le flux entrant tel que la valeur de la fraction volumique à l'extérieur soit fixée à 0,01 .

- A la frontière Est (côté fleuve), une condition de débit est imposée en considérant de l'eau clair $\left(\alpha_{s}=0\right)$.

Le calcul de la vitesse sur la maille au-dessus du fond, est assumé par une loi logarithmique de paroi proposée par Rodi [5]. Pour prendre en compte les phénomènes de turbulence, nous utilisons un modèle de viscosité turbulente basé sur le modèle de longueur de mélange (formule de Prandtl [6]) où la formulation de la longueur de mélange est celle proposée par Escudier [7].

\section{Résolution numérique}

Les détails concernant la résolution du système précédemment décrit sont présentés par Barbry et al. [1] [8]. Les auteurs ont développé un modèle bidimensionnel vertical $\mathrm{X} / \mathrm{Z}$ à deux phases pour le transport des particules fines en estuaire. Un maillage de type Eulérienadaptatif a été mis en place pour prendre en compte les évolutions de la surface libre (Guillou et al. [9]). Un algorithme à pas fractionnaire est utilisé pour résoudre le problème couplé à une formulation de type volumes finis. Une attention particulière a été portée au calcul de $\alpha_{\mathrm{s}}$ par l'utilisation d'un schéma strictement conservatif.

\section{Application du modèle à l'estuaire de la Seine}

L'estuaire de la Seine se jette dans la mer de la Manche au niveau du Havre. Avec un marnage moyen de 7 mètres à l'embouchure, il est de type macrotidal. Les effets dus au frottement y sont dominants par rapport aux effets de convergence, c'est un estuaire hyposynchrone : la marée est amortie progressivement de l'embouchure jusqu'au fleuve. Les apports fluviaux en matière en suspension à Poses sont en moyenne de 0.5 million de tonnes par an (de 0.2 à 1 million de tonnes par an) [10]. Le débit fluvial moyen est de $480 \mathrm{~m}^{3} / \mathrm{s}$ (compris entre 100 et $2000 \mathrm{~m}^{3} / \mathrm{s}$ suivant les saisons). 
La concentration dans le bouchon vaseux est de l'ordre de $2 \mathrm{~g} / \mathrm{L}$, sa position fluctue avec la marée et les variations du débit fluvial.

\subsection{Domaine et condition de simulation}

Le domaine modélisé s'étend sur 160 kilomètres du musoir (extrémités des digues submersibles) au barrage de Poses. La bathymétrie utilisée provient de la carte SHOM 6796. Le maillage (figure 1) est composé de 161 mailles dans la direction longitudinale et de 21 mailles dans la direction verticale, avec un raffinement au fond.

Les sédiments sont initialement introduits sous la forme d'une concentration moyenne imposée $(0.4 \mathrm{~g} / \mathrm{L})$ sur tout le domaine, le rayon des particules solides a été fixé à $10^{-5} \mathrm{~m}$ en accord avec les mesures in-situ. Le débit fluvial est de $500 \mathrm{~m}^{3} / \mathrm{s}$. Les résultats avec sédiments sont obtenus après 10 jours de simulation.

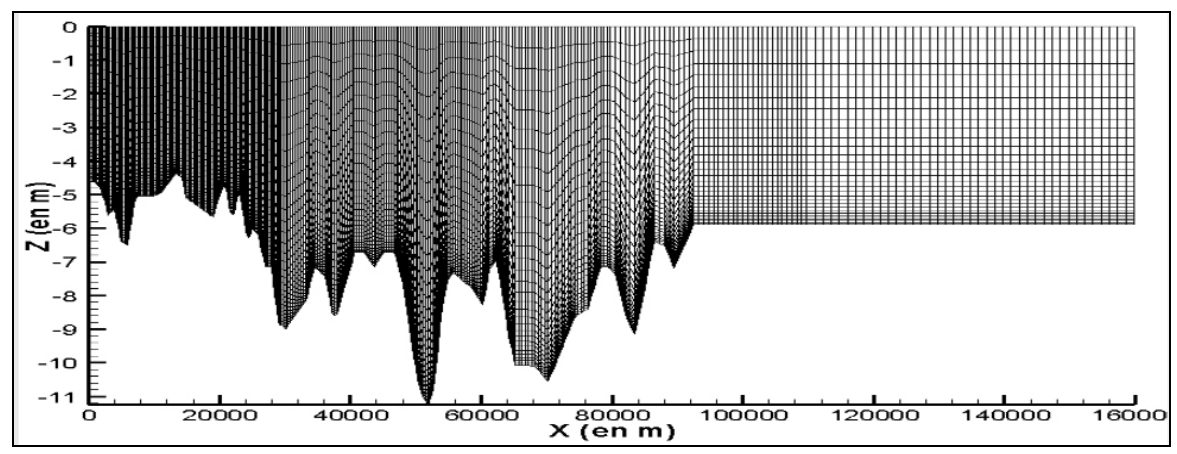

Figure 1:Maillage de l'estuaire (161 x 21)

\subsection{Résultats et comparaisons}

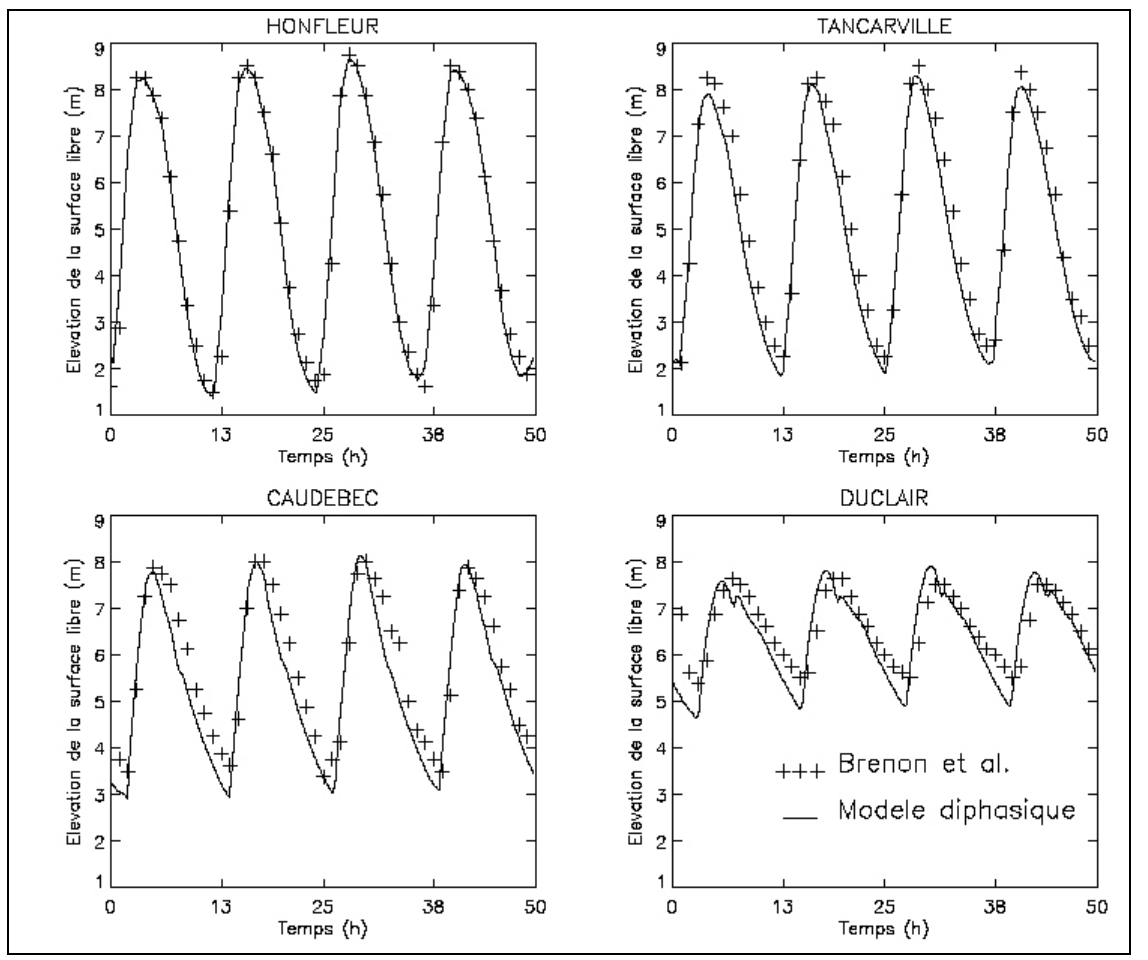

Figure 2: Hauteurs d'eau en différents points de sondage. 
Les hauteurs de surface libre (figure 2) et les vitesses moyennées (figure 3) sur la profondeur sont comparés aux résultats de Brenon [11] sur une période de 50 heures et en différents points de sondage (Honfleur, Tancarville, Caudebec et Duclair).

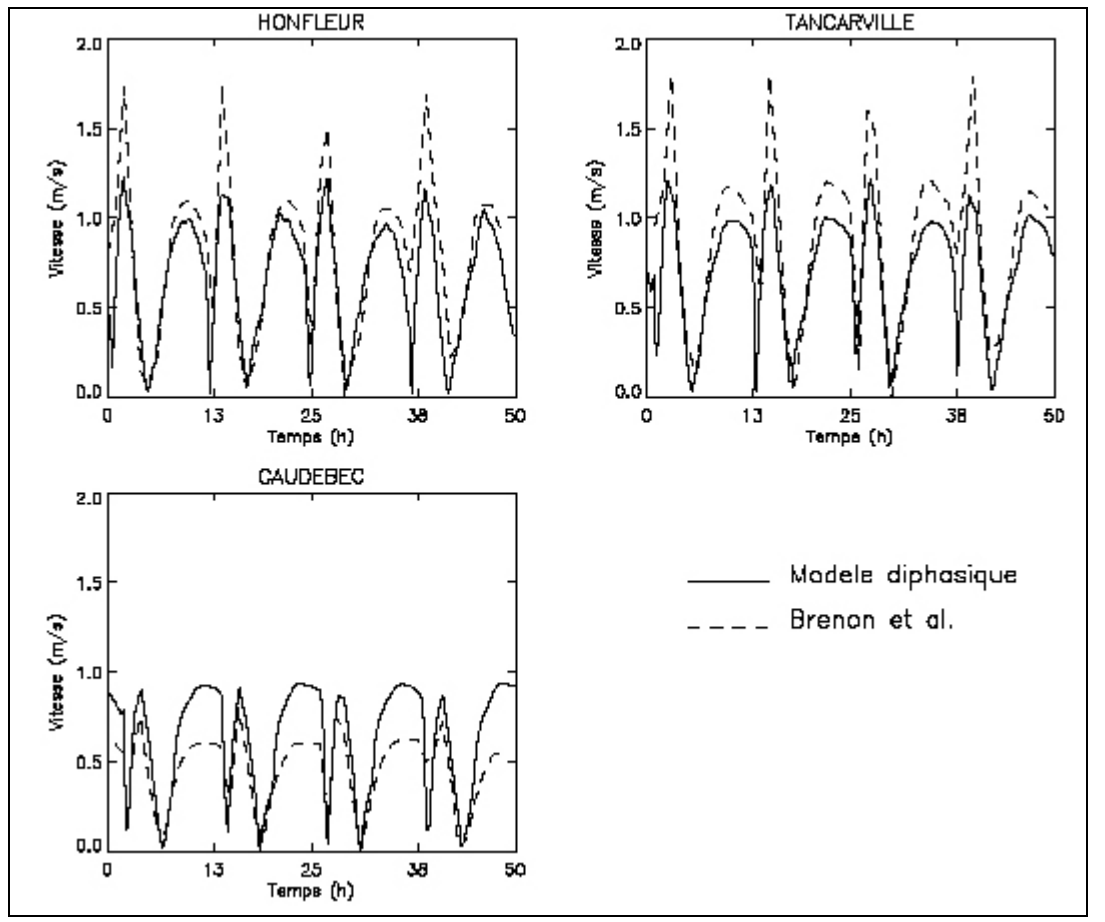

Figure 3:Vitesse du courant moyennée sur la profondeur en différents points de sondage

La figure 2 montre la transformation de l'onde de marée au cours de sa propagation dans l'estuaire. La courbe de l'élévation de la surface libre est symétrique à Honfleur pour devenir asymétrique à Duclair : la durée du flot est de plus en plus courte alors que celle du jusant est de plus en plus longue. A Duclair, le flot dure 4 heures alors que le jusant dure 8 heures. On retrouve cette asymétrie sur la vitesse du courant (figure 3). Le caractère hyposynchrone de l'estuaire est correctement reproduit par le modèle. Les vitesses de flot sont supérieures à celles de jusant. Cet effet s'atténue vers l'amont, ainsi à Duclair la vitesse de jusant égale celle de flot. Des différences sur l'amplitude de la vitesse apparaissent entre nos résultats et ceux de Brenon [11]. Etant donné l'absence de mesure, nous sommes dans l'incapacité de discuter ces différences. Par ailleurs, on note que la nature de ces deux modèles diffère. Notre modèle est intégré sur la largeur, ce qui est tout à fait acceptable pour la Seine où les variations latérales sont faibles. Le modèle de Brenon quand à lui est intégré sur la profondeur. De ce fait, les résultats sont difficilement comparables.

La figure 4 montre les isocontours de turbidité, heure par heure, sur un cycle de marée. Les sédiments initialement en suspension forment un bouchon vaseux qui se déplace avec la marée. A PM (Le Havre), le bouchon vaseux se situe dans sa position la plus amont. Le dépôt est maximal. De 1 heure à 6 heures après la PM, les sédiments se déplacent vers l'aval sur une distance d'environ 15 kilomètres. A BM le bouchon vaseux est dans sa position la plus aval. Le déplacement longitudinal du bouchon vaseux et sa localisation sont en accord avec les mesures in situ réalisées lors du Schéma d'Aptitude et d'Utilisation de la Mer [10]. 


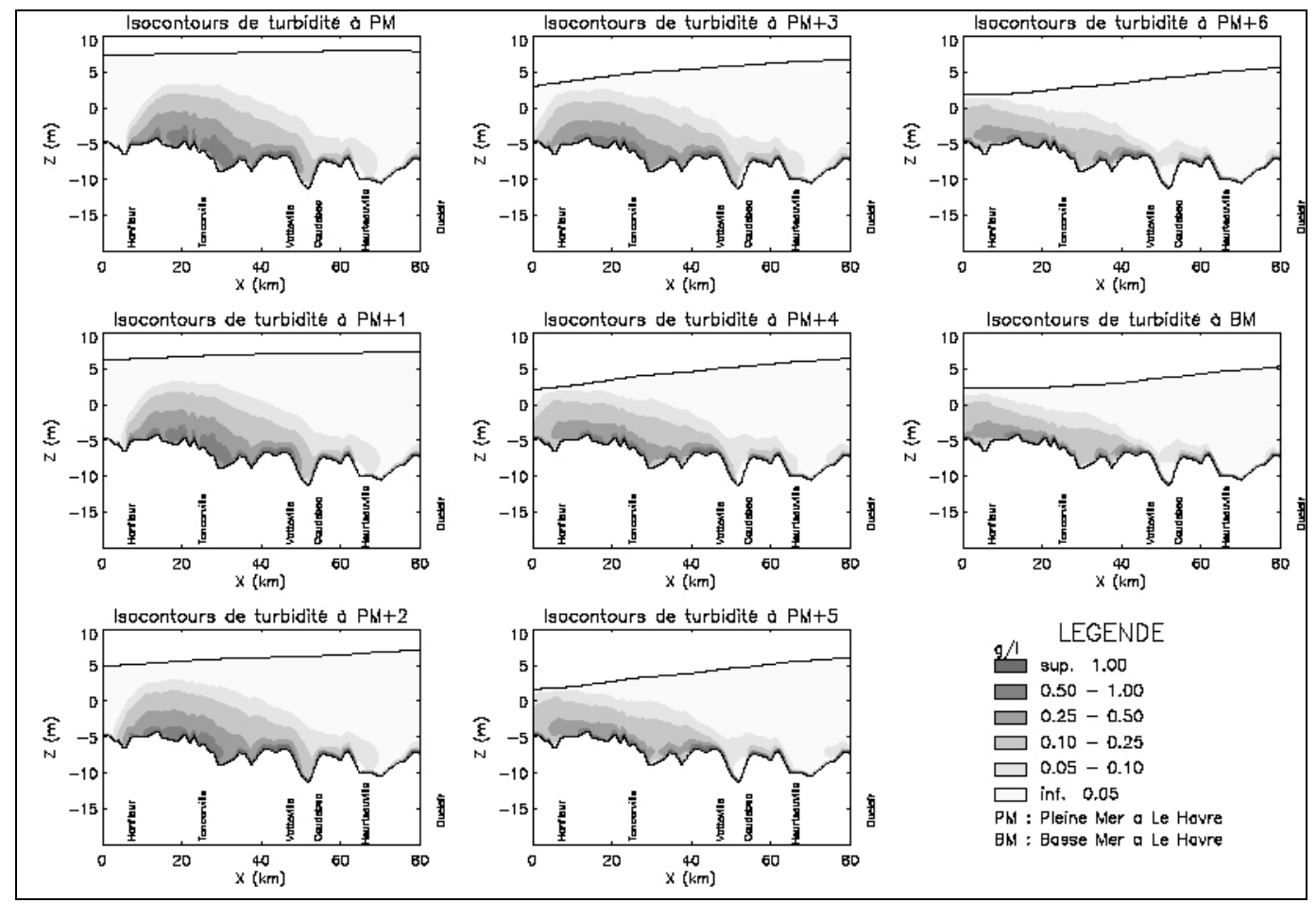

Figure 4:Evolution de la turbidité au cours d'un cycle de marée

\section{Conclusion}

Un modèle diphasique 2DV a été utilisé pour la simulation du bouchon vaseux dans l'estuaire de la Seine. La dynamique sédimentaire est satisfaisante, l'ordre de grandeur du déplacement du bouchon vaseux est correct ainsi que son comportement au cours d'un cycle de marée.

Cette étude montre l'applicabilité d'un modèle multiphasique à un estuaire réel. Une comparaison avec des mesures est nécessaire afin de réaliser une calibration plus fine de notre modèle.

\section{Références :}

1.Barbry N., Guillou S. and Nguyen K. D. (2000), Une approche diphasique pour le calcul du transport sédimentaire en milieux estuariens. Compte Rendu de l'Académie des Sciences (série II B) 328:pages 793 -- 799.

2.Lyczkowski R. W., Bouillard J. and Gidaspow D. (1989), Porosity distributions in a fluidised bed with an immersed obstacle. AIChE J. 35:pages 908 -- 922.

3.Graham A. L. (1981), On the viscosity of suspensions of solide spheres. Applied Science Research 37:pages 275 -- 286.

4.Drew D. A. and Lahey R. T. (1993), Analytical modelling of multiphase-flow, Particulate two-phase flows chapter 16, pages 509 -- 566. Butterworth-Heinemann series on chemical engineering. Rocco.

5.Rodi W. (1980), Turbulence models and their application in hydraulics. Monograph.

6.Prandtl L. (1925), Uber die ausgebildete turbulentz. ZAMM.

7.Escudier M. P. (1966), The distribution of mixing length in turbulent flow near walls. Heat transfer section Report TWF/TN/1, Imperial College.

8.Barbry N. (2000), Modélisation du transport sédimentaire en milieux estuariens selon une approche diphasique (fluide-particule) $\}$. PhD thesis, Université de Caen.

9.Guillou S., Barbry N. et Nguyen K. D. (2000), Calcul numérique des ondes de surface par une méthode de projection et un maillage eulèrien adaptatif. Compte Rendu de l'Académie des Sciences (série II B) 328:pages 875 à 881. 


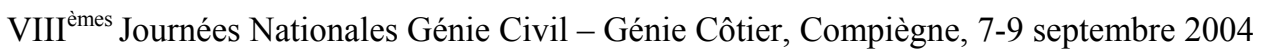

10.Avoine J. (1981), L'estuaire de la Seine : sédiments et dynamique sédimentaire. PhD thesis, Université de Caen.

11.Brenon I. (1997), Modélisation de la dynamique des sédiments fins dans l'estuaire de la Seine. PhD thesis, Université de Bretagne Occidentale. 